\title{
Precocious neurons may stunt brain growth in rare form of autism
}

\section{BY PETER HESS}

5 OCTOBER 2021

Mice with a mutated copy of MYT1L, a leading autism candidate gene, have unusually small brains and many other physical and behavioral traits mirroring those seen in people with similar mutations, according to a study published today in Neuron.

The mice represent the first model of MYT1 $\mathrm{L}$ syndrome, a rare genetic condition marked by autism, intellectual disability, attention deficit hyperactivity disorder (ADHD), obesity and microcephaly, or a smaller-than-average head.

"Generating a mouse line is always a gamble," says lead investigator Joseph Dougherty, associate professor of genetics and psychiatry at Washington University in St. Louis, Missouri. "The stars really aligned for us."

MYT1L encodes a transcription factor, a type of protein that influences gene expression. But few studies have explored how mutations in the gene lead to the traits seen in people, partly because there are likely fewer than 100 cases worldwide.

Dougherty and his colleagues used CRISPR to engineer mice with a MYT1L mutation that resembles one identified in an autistic person. The mice have neurons that mature earlier than expected, which could help explain the traits seen in people.

As the first mouse model of MYT1L mutations, "this is a landmark piece of work, and is certainly promising for fundamental science exploration and as a preclinical model," says Charis Eng, chair of the Cleveland Clinic's Genomic Medicine Institute in Ohio, who was not involved in the work.

\section{Physical similarities:}

Physical examinations of the MYT1L mice suggested to the team early on that they were on the 


\section{Spectrum | Autism Research News}

https://www.spectrumnews.org

right track. Like most people with the mutation, the mice are overweight and, like some people with the mutation, have atypically curved digits. Behavioral assays revealed further similarities.

The mice are also hyperactive, says Jiayang Chen, a doctoral student in Dougherty's lab who worked on the study. "This is a very robust phenotype that we saw across different generations and across different behavior assays."

Multiple tests suggested that the MYT1L mice are less sociable than wildtype mice: They show a limited degree of interest in other mice and spend less time investigating them than controls do. In one test, a mouse had to poke a button with its nose to open a window into another mouse's chamber for a brief chance at interaction. The MYT1L mice - and especially the males - poked the button but tended not to approach the window when it opened.

Animal models do not allow for one-to-one comparisons with people, Dougherty says. For instance, whereas autistic people may not make frequent eye contact with other people, mouse models of the condition may sniff each other less often or less enthusiastically than usual.

But given that mice are so different from people, it is "heartening that this model does recapitulate a lot of the human-relevant phenotype," Eng says.

The goal of studying the MYT1L mice is to identify which brain functions are altered when MYT1L is mutated, and then to study those circuits in people.

The new results open the door to investigating therapies that could ease some of these traits for people with MYT1L syndrome, Dougherty says. "If you have a good model, you can work on reversing what you see."

\section{Precocious neurons:}

Brain scans showed that MYT1L mice have reduced overall brain volume and white matter, the bundles of nerve fibers wrapped in myelin that connect neurons from different brain regions.

The mutation ramps up the expression of genes involved in brain growth but dampens that of genes that encourage neurons to divide and make more brain cells, according to comparisons of RNA sequencing data from embryonic and adult mouse brains. Many of these dysregulated genes are linked to autism and intellectual disability.

Instead of dividing and multiplying, causing the brain to grow, the mice's brain cells tend to mature too soon and differentiate into their final form early, cell staining of embryonic mouse brain samples revealed. The result is microcephaly.

It is not totally clear which neuron populations are missing in the smaller brains, though, says 


\section{Spectrum | Autism Research News}

https://www.spectrumnews.org

Brady Maher, lead investigator at the Lieber Institute for Brain Development in Baltimore, Maryland, who was not involved in the study. "It could be that all types of neurons are generally missing, but it could also be that a specific type of neuron is missing."

Notably, the genes with altered expression in the MYT1L mice overlap with those that are altered in other mouse models of autism. For instance, some mutations in CHD8, which regulates other autism-linked genes, result in macrocephaly, or a larger-than-average head.

"We found very significant overlap between the two," Chen says. The brain-growth genes that CHD8 turns up are turned down in the MYT1L mice, and vice versa.

Beyond cell maturation, MYT1L is involved in the normal functioning of myelin in white matter, an important element for neuronal connectivity. Its disruption can lead to some of the neural circuit dysfunctions observed in autism, says Jun Hee Kim, associate professor of cellular and integrative physiology at the University of Texas Health Science Center in San Antonio, who was not involved in the study. "This study shows that MYT1L is critical for the early development of neural circuits."

Dougherty and his colleagues next plan to generate conditional knockout mice - mice missing both copies of MYT1L just in specific brain regions - to home in on exactly which brain circuits are affected by mutations in or loss of the gene.

Cite this article: https://doi.org/10.53053/QFGG4751 